\title{
Discussion on Detection Method of Continuous Compaction Control Technology in Filling Engineering
}

\author{
Yu Qi ${ }^{1, ~ *, ~ J i a n g ~ H u i h u a n g ~}{ }^{2,3}$, Gao Mingxian $^{3}$, Xiang Weiguo $^{2}$, Yan Xiaoxia ${ }^{3}, W_{\text {u Longliang }}^{2}$ \\ ${ }^{1}$ Bureau Public Work of Shenzhen Municipality, Shenzhen, China \\ ${ }^{2}$ China Academy of Railway Sciences Corporation Limited, Beijing, China \\ ${ }^{3}$ Shenzhen Research and Design Institute, China Academy of Railway Sciences Corporation Limited, Shenzhen, China
}

\section{Email address:}

ramlee@126.com (Yu Qi),651825979@qq.com (Jiang Huihuang),1564322199@qq.com(Gao Mingxian)

${ }^{*}$ Corresponding author

\section{To cite this article:}

Yu Qi, Jiang Huihuang, Gao Mingxian, Xiang Weiguo, Yan Xiaoxia, Wu Longliang. Discussion on Detection Method of Continuous Compaction Control Technology in Filling Engineering. American Journal of Civil Engineering. Vol. 7, No. 4, 2019, pp. 113-120. doi: 10.11648/j.ajce.20190704.15

Received: September 8, 2019; Accepted: October 21, 2019; Published: October 23, 2019

\begin{abstract}
Compared with the traditional sampling quality detection method, the continuous compaction control technology has significant advantages in real time, full range and comprehensiveness. Therefore, this technology has gradually been widely used in the filling project. However, there are more than ten kinds of continuous compaction control methods, and the applicability of each method is different. Therefore, in order to promote the continuous application of continuous compaction control technology in China. The basic principles of various testing methods for continuous compaction control technology of filling engineering are summarized. The existing continuous compaction control technology testing methods are divided into four categories: (1) compaction method; (2) stiffness/modulus Method; (3) kinetic method; (4) energy method. The calculation process and supporting equipment of each detection method are introduced respectively. The applicability of various methods is analyzed based on the basic theory of various methods. The applicable scope and application suggestions of each detection method are proposed. The results show that the compaction method and energy method can be applied to fine-grained fillers, and the stiffness/modulus method and kinetic method can be applied to coarse-grained fillers and asphalt mixtures. According to the specific engineering conditions, the selection of suitable testing methods for continuous compaction control can obtain satisfactory application results.
\end{abstract}

Keywords: Continuous Compaction Control, Detection Method, Applicability, Compaction Method

\section{Introduction}

The filling project refers to the collective name of the geotechnical structure that is built and crushed according to certain requirements, covering the railway, highway, airport, dam, municipal and other fields [1]. According to the "Thirteenth Five-Year Plan" of the Ministry of Communications, China's filling project will enter a large-scale rapid construction phase. Compaction quality testing is the most important means to ensure the quality of filling projects. Compaction quality testing methods and testing indicators play a key role in quality control [2-4]. However, the current point detection methods are poorly representative, lack of process detection and key detection, inability to evaluate discrete large fillers, and quality control that is difficult to achieve compaction uniformity $[1,3]$. In response to the limitations of the point detection method, Sweden first proposed the detection method of continuous compaction control in the 1970s [5]. The method evaluates the compaction quality of the filling structure by installing a sensor on the vibrating wheel of the vibratory roller and the dynamic response signal reflected by the sensor. The working principle is shown in Figure 1.

With the advancement of science and technology, continuous compaction control technology has developed rapidly. Among them, the detection method of continuous compaction control technology has received extensive attention as the core content of this technology. At present, 
there are more than ten kinds of continuous compaction control technology detection methods. However, a large number of engineering practices have shown that [6-9], the existing detection methods have a certain scope of application, and improper selection of detection methods and equipment may directly lead to failure in the application of continuous compaction control technology. It can be seen that it is especially necessary and urgent to have an in-depth understanding of the basic principles of various testing methods and the equipment used. In view of this, this paper summarizes the existing continuous compaction control detection methods for filling projects, and on this basis, discusses the applicability of various methods, and provides reference for the correct application of continuous compaction control technology.

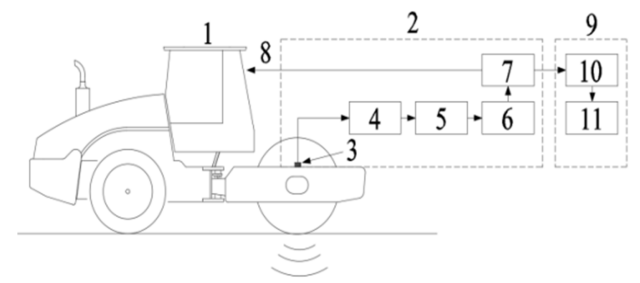

Figure 1. The basic principle of continuous compaction control.

(1-loading equipment; 2-detection equipment; 3-sensors; 4-signal conditioning; 5-data collection; 6-analytical processing; 7-display; 8-feedback control; 9-information management system;; 11-Remote Information Management)

\section{Introduction to Detection Methods}

The current continuous control methods can be divided into four categories according to different principles: (1) Compaction meter method, which provides empirical indicators based on the spectrum of vibration signals, such as the harmonic ratio of CMV in Sweden [5-7, 10], Japan's harmonic ratio derivative index CCV [5, 11]; (2) stiffness / modulus method, based on the mechanical principle, the method has a clear physical meaning, such as the Swiss stiffness coefficient [5, 12], Germany's dynamic modulus [5, 12]; (3) dynamics method, based on the dynamics principle proposed resistance index, such as China's vibration compaction value [1, 3, 13]; (4) energy Method [5], which is based on the energy required to overcome the resistance to motion when the roller is traveling, such as the energy index in the United States. The various testing methods are described in detail below.

\subsection{Compaction Method}

\subsubsection{Basic Principles and Equipment of CMV}

CMV is a continuous compaction control index jointly developed by GEO-DYNAMIK and DYNAPAC in the 1970s and commercialized in the 1980s. The method evaluates the compaction state of the filling body by discriminating the degree of distortion of the vibration wheel response signal of the vibratory roller. The spectral characteristics are shown in Figure 2. In order to quantitatively analyze the degree of distortion of the vibration wheel response signal, the developer defines a CMV that continuously evaluates the degree of waveform distortion through spectrum analysis, that is, the compaction value. The CMV is obtained by multiplying the ratio of the second harmonic amplitude and the first harmonic amplitude in the frequency domain by the vertical acceleration of the vibrating wheel by a coefficient, and the calculation formula is as shown in Equation 1. The CMV is determined by spectral analysis of the vertical acceleration of the vibrating wheel measured during two vibration cycles (see Figure 2), so the CMV is actually the calculated average of two cycles. CMV accuracy is controlled by the $1 \%$ distortion resolution of the accelerometer. According to Equation 1, the amount of change in CMV generated by $1 \%$ of acceleration per distortion is 3 .

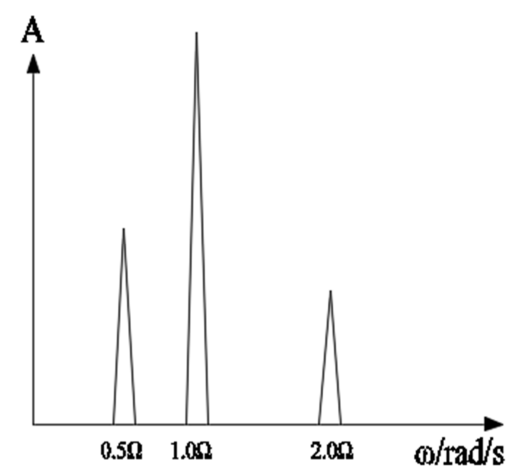

Figure 2. Vibration signal spectrum analysis.

$$
C M V=c \frac{A_{2 \Omega}}{A_{\Omega}}
$$

The continuous compaction control equipment developed based on the CMV index includes Dynapac's DCA compaction analyzer, Xugong Group's SMC-960A compactness measuring instrument, and China Water Resources and Hydropower Research Institute YS-1 compaction meter. The basic principles of various types of equipment are similar, mainly represented by DCA compaction analyzer. The DCA compaction analyzer can output CMV, data, amplitude, frequency, GPS coordinates and other data related to vibration compaction in a graphical format through data calculation and processing combined with GPS positioning technology, as shown in Figure 3. Dynapac uses a GPS (DGPS) differential receiver with recorded $X$ and $\mathrm{Y}$ coordinates with centimeter-level accuracy. Dynapac also used a better RTK receiver and satellite calibration via Omnistar, which enabled the DCA to record Z-coordinates (filling elevations). The DCA software enables real-time monitoring of all compacted data in local coordinates by local transformation between the World Geodetic System (WGS 84) and the local grid. The DCA produces one CMV per second, and the spatial resolution of the recorded data is primarily determined by the speed of the roller. Therefore, Dynapac recommends that the roller travel at a speed of no more than $1.0 \mathrm{~m} / \mathrm{s}$, which ensures that the spatial resolution of the $\mathrm{X}$-direction is no more than $1.0 \mathrm{~m}$ and the Y-direction resolution is the width of the vibrating wheel (usually $2.13 \mathrm{~m}$ ). 
The data recorded by the DCA can be exported from the field computer in a text file format for further analysis.

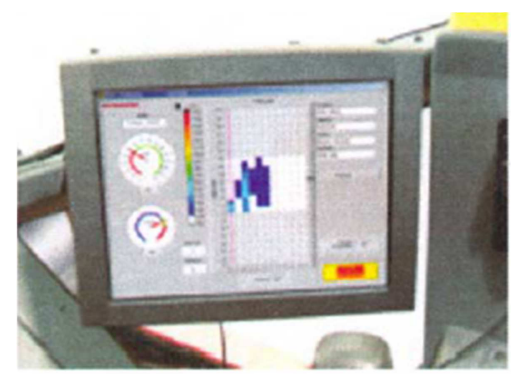

Figure 3. DCA type compaction analyzer output interface.

\subsubsection{Basic Principles and Equipment of CCV}

The CCV indicator is an extension of the CMV indicator, and its basic principle is the same as CMV. The difference is that CCV believes that the degree of compaction is not only related to the 1.0 and 2.0 times harmonic amplitude, but also to the $0.5,1.5,2.5$ and 3 harmonic amplitudes. The calculation principle of the $\mathrm{CCV}$ indicator is shown in Equation 2.

$$
C C V=\left[\frac{A_{1}+A_{3}+A_{4}+A_{5}+A_{6}}{A_{1}+A_{2}}\right] \times 100
$$

Where: A1-A6 represent the amplitudes of the 0.5, 1.0, 1.5, 2.0, 2.5, and 3.0 harmonics, respectively.

The continuous compaction control equipment developed based on the $\mathrm{CCV}$ index is represented by a compaction analyzer manufactured by Sakai. The basic principle of the device is consistent with DCA, with real-time monitoring, data recording and graphic output. The output interface of the compaction analyzer produced by Sakai is shown in Figure 4.

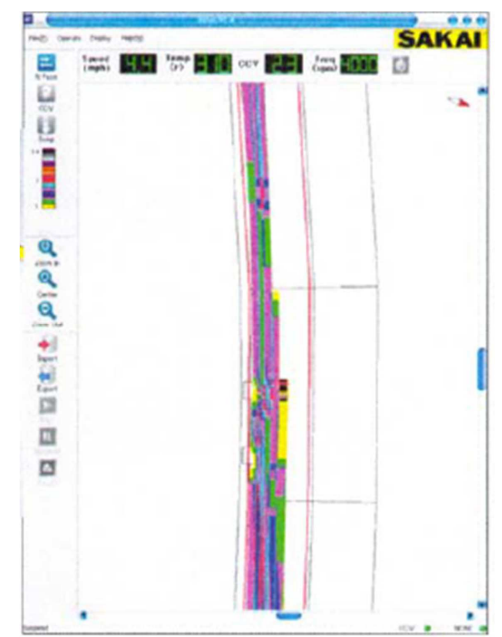

Figure 4. Interface of the output of the compaction analyzer.

\subsection{Stiffness / Modulus Method}

\subsubsection{Basic Principles and Equipment of Ks}

$\mathrm{Ks}$ is a stiffness coefficient index proposed by Ammann based on the principle of interaction between the vibrating wheel and the rolling problem. The index uses a two-degree-of-freedom model as shown in Figure 5.

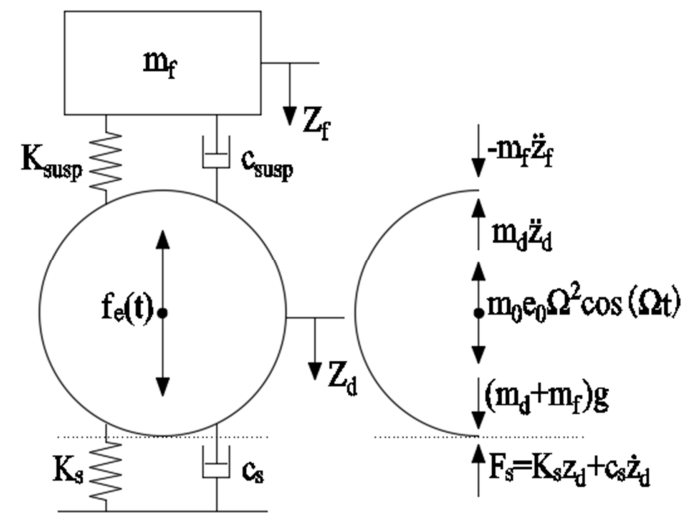

Figure 5. Ks calculation model.

In the model shown in Figure 5, the soil is represented by the Kelvin model, which is the excitation frequency of the roller; md and $\mathrm{mf}$ are the masses of the vibrating wheel and the frame respectively; $\mathrm{zd}$ and $\ddot{z}_{d}$ are the displacement and acceleration of the vibrating wheel, respectively; The eccentric moment in the vibrating wheel. It can be seen from Figure 5 that the contact force Fs of the vibrating wheel and the crushing body is composed of four parts: the vibrating wheel inertia, the frame inertia, the eccentric force and the roller distributing weight. The inertia and eccentric force of the drum are determined by measuring the vertical acceleration and the eccentric position of the vibrating wheel, while determining the displacement amplitude $z_{d}$ by integrating the acceleration peak. When the frame inertia and the roller distribution weight are ignored and the vertical velocity component of the eccentric block is zero (i.e., the eccentric block is at the lowest position), the calculation equation as shown in Equation 3 can be obtained. Therefore, Ks can be determined based on the measured acceleration and phase lag of the vibrating wheel. Ks is actually the ratio of the contact force to the maximum vertical displacement of the vibrating wheel when the vertical velocity component of the eccentric block is zero, as shown in Figure 6.

$$
K_{s}=\Omega^{2}\left[m_{d}+\frac{m_{0} e_{0} \cos (\varphi)}{z_{d}}\right]
$$

Where: $\varphi$ is the phase lag between the eccentric force and the vibration wheel displacement.

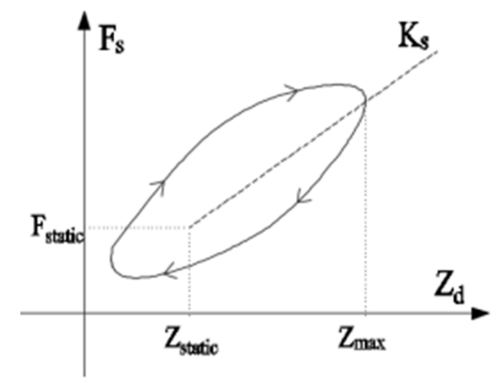

Figure 6. Ks calculation principle. 
The main manufacturers of continuous compaction control equipment based on the Ks index are Ammann and Case, which are represented by Ammann's Ace Plus system. The Ace Plus system calculates the stiffness factor of the compacted body every vibration. The accuracy of the measured data is as follows: $\Delta z_{d}$ is not more than $0.001 \mathrm{~mm}$; $\Delta \varphi$ is not more than $0.5^{\circ} ; \Delta \Omega$ is not more than $0.31 \mathrm{rad} / \mathrm{s}$. The Ace Plus software (see Figure 7) combines the Ks data with the $\mathrm{X}, \mathrm{Y}$, and $\mathrm{Z}$ coordinates acquired by the in-vehicle GPS device with a coordinate accuracy of less than $10 \mathrm{~cm}$. When there is no reference signal (such as a base station), the

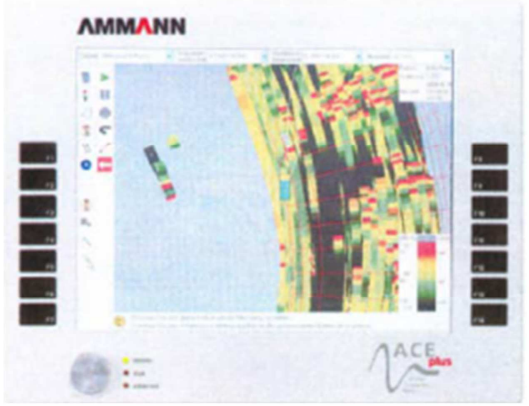

(a) Vehicle display interface accuracy of the system is within a few meters. The Ace Plus system calculates the metric for each vibration cycle and combines the GPS data to report the average at $1 \mathrm{~Hz}$. The system software is installed in the tablet computer, and outputs real-time vibration rolling parameters in a graphic format, as shown in Figure 7a. All recorded data can be downloaded from the onboard computer in a text file format via a USB memory stick to compact the quality assessment. It should be noted that Amman also provides a PC-based operating platform for remote monitoring (see Figure $7 b$ ), which can greatly improve project management efficiency.

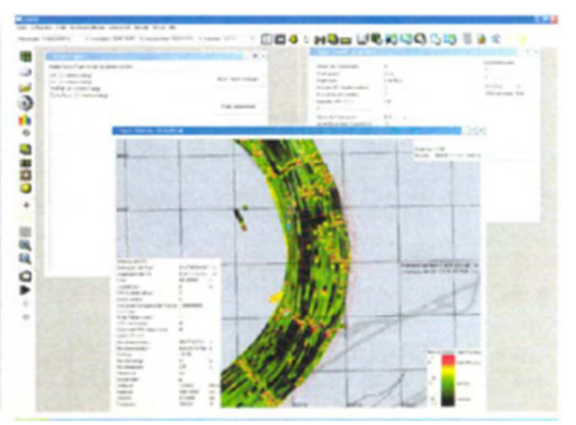

(b) Platform display interface

Figure 7. Ace Plus software output interface.

\subsubsection{Basic Principles and Equipment of Evib}

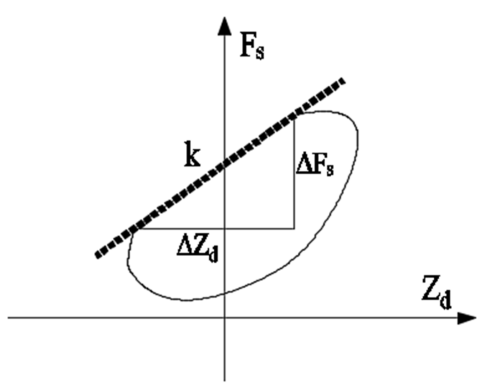

Figure 8. Secant stiffness K solution.

Evib is a dynamic modulus index proposed by Bomag based on vibration theory and elastic half-space theory. The index uses the same two-degree-of-freedom model as the stiffness coefficient index Ks. According to the mechanical equilibrium condition of the calculation model, the contact force Fs and the vertical displacement $\mathrm{zd}$ of the vibrating wheel can be solved, and the yield-disturbance curve of the compacted body is obtained by the combination of the data of

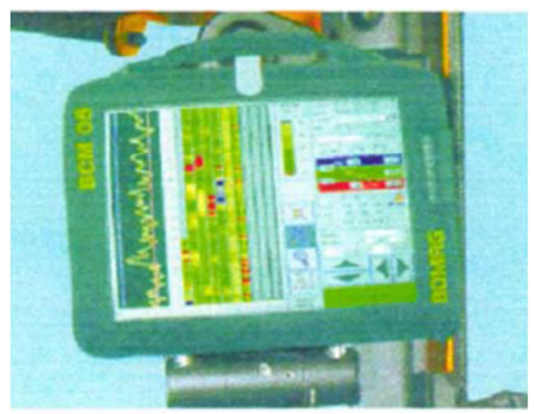

(a) Vehicle display interface and, as shown in Figure 8. To determine Evib, the secant stiffness is calculated from the compression portion of each Fs and $\mathrm{zd}$ cycle, and the obtained secant stiffness is linked to the dynamic modulus Evib. The dynamic modulus Evib can be obtained by using the theoretical solution of the rigid cylinder on the isotropic and uniform elastic half space proposed by Lundberg [5], as shown in Equation 4.

$$
z_{d}=\frac{2 \times\left(1-v^{2}\right)}{\pi \times E} \times \frac{F_{s}}{L} \times\left(1.8864+\ln \frac{L}{b}\right)
$$

among them

$$
b=\sqrt{\frac{16 \times R \times\left(1-v^{2}\right)}{\pi \times E \times L} \times F_{s}}
$$

Where: $\mathrm{L}$ is the vibration wheel width; $\mathrm{R}$ is the vibration wheel radius; $v$ is the Poisson's ratio; $\mathrm{E}$ is the Young's modulus.

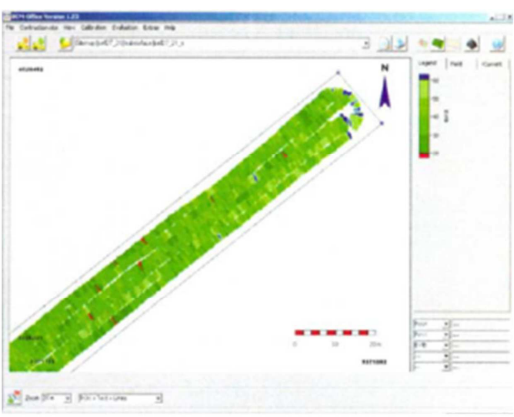

(b) Platform display interface

Figure 9. Vario Control system output interface. 
Based on the Evib index, the development of continuous compaction control equipment is represented by the Vario Control system developed by Bomag. The system mainly includes car tablet, mobile software and USB memory stick for data transmission. The data output interface of the system is shown in Figure 9. The Vario Control system accepts any GPS receiver that can provide GGA or PJK data via the RS232 interface. It reports and stores the Evib at the GPS sampling coordinates at a frequency of $10 \mathrm{~Hz}$. The accuracy of the Omnistar-corrected report is less than $5 \mathrm{~cm}$.

\subsection{Kinetic Methods}

The dynamic testing method of continuous compaction control technology is represented by the resistance method proposed in China. According to the dynamic principle of the interaction between the vibration wheel of the road roller and the subgrade structure, the relationship between the response index and the degree of compaction is established. The acceleration response index VCV is used as the continuous compaction control index [1]. The resistance of the filling structure is the reaction of the filling structure to the rolling machine, and the resistance can characterize the ability of the filling structure to resist deformation. Therefore, the strength and stiffness of the filling structure are indirectly known by resistance $[1,3,13]$. According to the dynamic principle, the mechanical vibration response signal is used to identify the resistance of the filling structure. When the parameters of the vibration machine are constant, the mechanical index reflecting the compaction state of the filling body can be established as shown in Equation 6.

$$
V C V=P \sin (\omega t)+M g-\eta M f(\ddot{u}, \omega)
$$

It can be seen from Equation 6 that VCV is only related to vibration signals and mechanical parameters, ie

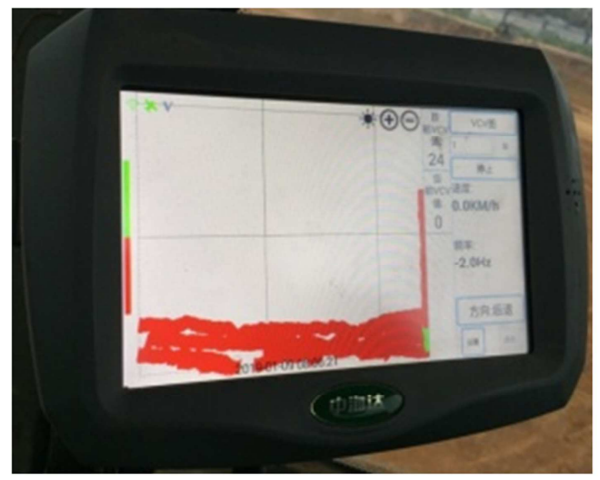

(a) Vehicle interface

$$
\begin{gathered}
V C V \sim F(M, P, f, u, \dot{u}, \ddot{u}) \\
\Delta V C V \sim \Delta F
\end{gathered}
$$

Where: $\mathrm{P}$ is the excitation force amplitude; $\omega$ is the eccentric block angular velocity; $M$ is the vibration wheel mass; $u, \dot{u}, \ddot{u}$ respectively the vibration wheel displacement, velocity and acceleration; $f$ is the excitation frequency; $\eta$ is the comprehensive correction function, is a dynamic quantity; $f(\ddot{u}, \omega)$ is a function or time series related to vibration wheel acceleration and vibration frequency.

It can be known from Equation 8 that for a rolling machine having a stable vibration parameter and stable performance, the VCV value of the crushed body can be obtained by continuously collecting the acceleration of the vibration wheel. In practical applications, it is not necessary to solve the absolute value of VCV, and it can be established by considering the factors related to $\mathrm{VCV}$ value and the routine detection indicators on site [1].

The continuous compaction control system based on VCV index mainly includes CCC-800 system developed by China Railway Science Research Institute and CPMS system developed by Southwest Jiaotong University. The continuous compaction control equipment based on VCV indicator is represented by the intelligent compaction equipment of Beidou roller, which is represented by Guangzhou Zhonghaida Satellite Navigation Technology Co., Ltd. Zhonghaida continuous compaction equipment mainly includes reference station, vehicle display terminal, mobile software and data acquisition box for storage. The equipment is equipped with Beidou engineering receiver and supports multi-star multi-frequency. The corrected positioning accuracy is centimeter. The display interface of the intelligent compaction device of Beidou roller is shown in Figure 10.

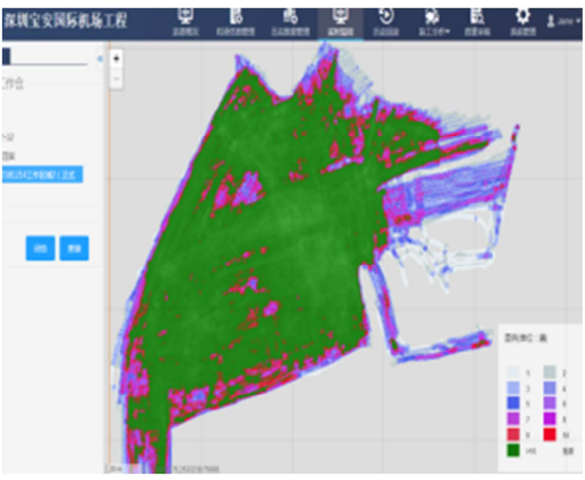

(b) Platform interface

Figure 10. Haida compaction equipment output interface.

\subsection{Energy Method}

The continuous compaction control detection index based on the energy principle mainly refers to the energy index MDP proposed by Caterpillar. The basic principle of the energy method is to study the interaction between the vehicle and the ground. The concept of rolling resistance and sinking is used to determine the stress acting on the drum and the energy required to overcome the motion resistance to characterize the compaction of the filling body.. The MDP calculation diagram is shown in Figure 11, and the calculation formula is as shown in Equation 9. 


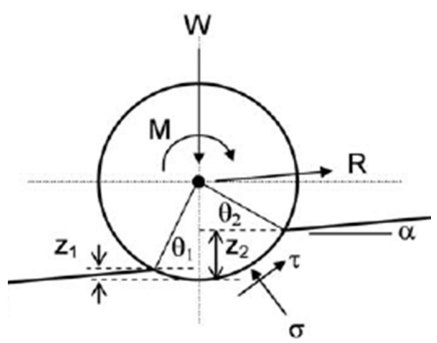

Figure 11. MDP calculation diagram.

$$
M D P=P_{g}-W V\left(\sin \theta+\frac{a}{g}\right)-(m V+b)
$$

Where: $P_{g}$ is the total power required to move the machine; $\mathrm{W}$ is the weight of the vibrating wheel; $\mathrm{a}$ is the acceleration of the machine; $g$ is the acceleration of gravity; $\theta$ is the angle of inclination; $\mathrm{V}$ is the speed of the vibrating wheel; $\mathrm{m}$ and $\mathrm{b}$ are the internal loss factor of the machine.

The second and third terms in Equation 9 represent the loss energy associated with the slope and the machine power associated with the internal machine loss, respectively. Before using MDP, the calculation parameters in Equation 9 are determined by field calibration, and the value of MDP at the calibration surface is set to zero, and the degree of compaction is judged by comparing the size of the MDP. Typically, a positive value for MDP indicates a relatively looser degree of compaction than a calibrated surface, while a negative value for MDP indicates a more compact degree of compaction (ie, a smaller amount of drum sinking).

The continuous compaction control system based on the MDP index is mainly represented by the Compaction Viewer system developed by Caterpillar, as shown in Figure 12. The Compaction Viewer system features data acquisition and satellite positioning to display position and compaction measurements in real time. The system uses GPS positioning technology with a horizontal accuracy of $\pm 10 \mathrm{~mm}$ and a vertical surface accuracy of $\pm 20 \mathrm{~mm}$. The system can realize visual monitoring of data on a PC.

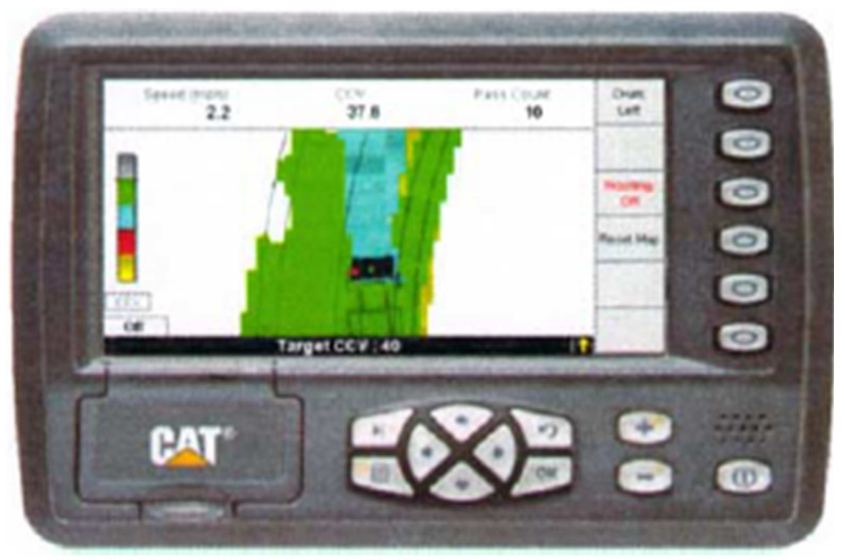

Figure 12. Compaction Viewer system output interface.

\section{Applicability Discussion}

With the maturity of continuous compaction control technology testing methods, countries and regions in the world have successively formulated corresponding technical standards and norms [14-16], and the technology has been stipulated in the relevant project contract terms. The continuous compaction technology has been well promoted and applied in engineering practice. The Cologne-Rheinhe high-speed railway Shinkansen project in Germany, the asphalt pavement compaction project in the Raleigh area of the United States, the Zoucheng section of the Beijing-Shanghai high-speed railway in China, the Bottom section of the Shanghai-Kunming high-speed passenger line, the Minjiang section, and the Shiji railway passenger transport The continuous subgrade compaction control technology has been successfully applied to the project of the base station of the East Plain Station and the TJ-10 section of the Beijing-Shenyang Passenger Dedicated Line, and has achieved good results. However, the Minnesota Expressway Project in the United States, the Shenyang-Dandong Expressway Project in China, the Harbin-Dalian Passenger Dedicated Line Project, and the Lanxin Railway Project are applied because there is no good correlation between the continuous compaction test index and the compaction quality. failure. It can be seen that there are both successful experiences and many failed cases in practice. Analysis of the reasons can be seen, although the current continuous compaction control technology detection methods are more, but there are certain scope of application. For a wide range of engineering conditions, the ability to choose the right test method will directly affect the successful application of continuous compaction control technology. According to the basic principle of each detection method, the applicability of each method obtained by the analysis is as follows.

\subsection{Applicability of the Compaction Method}

The compaction gauge index evaluates the compaction state based on the degree of distortion of the response signal, and the main factor affecting the waveform distortion is the first harmonic component $[1,5]$. It is known from the vibration theory that the strength of the interaction between the road roller and the pressed structure determines the spectral composition of the response signal, which is the root cause of harmonic generation [5]. In fact, the contact of the roller with the packing is uncoupled, and the vibrating wheel is usually a nonlinear vibration. This nonlinear vibration characteristic is more pronounced especially in the case of coarse aggregates. Linear vibration generally only has a simple frequency doubling component, and nonlinear vibration is very complicated in the frequency domain. Therefore, the compaction method is often only applicable to the case of linear vibration, that is, rolling fine particle working conditions.

\subsection{Applicability of the Stiffness/Modulus Method}

Based on the mechanical principle of Switzerland's stiffness coefficient Ks, Germany's dynamic modulus Evib is 
mainly based on the interaction between the vibratory roller and the filling body, using the classical mechanics theory for complex derivation. The mechanical method characterized by vibration modulus requires that the vibration wheel displacement of the vibratory roller is coordinated with the deformation of the filling body (ie, close contact, no bounce phenomenon), and the actual vibratory roller in reality is difficult to meet the requirements. Such methods assume more conditions and often require a dedicated vibratory roller to be better applied.

\subsection{Applicability of the Kinetic Method}

According to the basic principle of $\mathrm{VCV}$, in order to make the linear relationship between the reaction force and the acceleration, the method usually does not consider the influence of the damping of the filling structure in practical application. Therefore, VCV is theoretically suitable for coarse-grained soils with relatively small viscosity coefficients [1, 13], and the applicability of fine-grained materials with non-negligible viscosity coefficient or damping coefficient needs further verification and research.

\subsection{Applicability of the Energy Method}

According to the principle of energy law, the basic assumption of its application is that there is no relative sliding between the vibrating wheel and the compacting body, and the difference between the corrected surface stiffness and the stiffness of the detecting surface must not be too large. In fact, it is difficult to ensure the close contact between the vibrating wheel and the crushing body without relative sliding in real time. The stiffness of the detecting surface also changes with the increase of the number of rolling passes, and it is difficult to satisfy the application conditions one by one. In fact, in the process of vibration compaction, the vibration wheel is not coupled with the surface of the filling body more than $50 \%$, especially for the coarse filler with large elastic modulus, the phenomenon of "jumping" is more obvious. Therefore, the energy method based on the principle of motion resistance is less applied, and few successful application examples are carried out under static rolling conditions. According to the 2016 edition of the European Union's continuous compaction control technical specifications, this method has not been applied.

Regardless of the detection method used, there are common problems such as the inconsistency between the continuous compaction detection range and the conventional quality inspection range, the influence of the filler water content and the underlying layer stiffness on the continuous detection results. Therefore, the applicability of various continuous compaction control detection methods is affected to varying degrees. By analyzing the basic principles of various methods and referring to the collected field test results of 35 engineering examples and research groups around the world, the selection suggestions for each method as shown in Table 1 were obtained.

Table 1. Recommendations for testing methods.

\begin{tabular}{|c|c|c|c|}
\hline Test & method & Filler & properties \\
\hline Compaction method & $\begin{array}{l}\text { Fine pellet } \\
\text { Coarse grain } \\
\text { Asphalt }\end{array}$ & $\begin{array}{l}\text { good } \\
\text { Not applicable } \\
\text { good }\end{array}$ & $\begin{array}{l}\text { Recommended for fine-grained materials, attention should be paid to the } \\
\text { control of water content of the filler }\end{array}$ \\
\hline Stiffness/modulus method & $\begin{array}{l}\text { Fine pellet } \\
\text { Coarse grain } \\
\text { Asphalt }\end{array}$ & $\begin{array}{l}\text { general } \\
\text { good } \\
\text { good }\end{array}$ & $\begin{array}{l}\text { Recommended for coarse-grained materials, pay attention to the } \\
\text { difference in stiffness of the underlying layer }\end{array}$ \\
\hline Dynamic method & $\begin{array}{l}\text { Fine pellet } \\
\text { Coarse grain } \\
\text { Asphalt }\end{array}$ & $\begin{array}{l}\text { general } \\
\text { good } \\
\text { good }\end{array}$ & $\begin{array}{l}\text { Can be used in the case of fine pellets, but special attention should be } \\
\text { paid to the moisture content control of the filler. Recommended for coarse } \\
\text { and mixed materials }\end{array}$ \\
\hline Energy method & $\begin{array}{l}\text { Fine pellet } \\
\text { Coarse grain } \\
\text { Asphalt }\end{array}$ & $\begin{array}{l}\text { good } \\
\text { Not applicable } \\
\text { general }\end{array}$ & Less applied, recommended for fines \\
\hline
\end{tabular}

In summary, the current continuous compaction technology detection methods have a certain scope of application, and can not be blindly adopted. In practical applications, it is necessary to select the detection method reasonably for the engineering characteristics. Practice shows that $[5-9,13]$, theory combined with field trials and drawing on similar engineering experience is an effective way to promote the successful application of this technology.

\section{Conclusion}

By summarizing the basic principles of various continuous compaction control detection methods and supporting application equipment, the main conclusions are as follows:

(1) The compaction method and the energy method estimate the degree of compaction of the filling body by the degree of distortion of the vibration signal, which is essentially an empirical method. The stiffness/modulus method and the dynamic method are based on the mechanical principle to solve the displacement and force of the vibration, so as to indirectly evaluate the compaction quality of the filling body, so it has a good theoretical basis and is a more reasonable theoretical calculation method.

(2) The compaction method and the energy method can be well applied to the fine-grained filler, and the stiffness/modulus method and the kinetic method can be suitably applied to the coarse-grain filler and the asphalt mixture.

(3) The current continuous compaction control technology 
detection methods generally have problems such as poor applicability of fillers with different physical and mechanical properties. For the specific engineering conditions, comprehensive selection of appropriate detection methods for continuous compaction control can obtain satisfactory application results.

\section{Acknowledgements}

This paper is supported by the technical research topic THZQ-033-2018 of Shenzhen Construction Industry Department.

\section{References}

[1] Xu Guanghui. Dynamic principle and engineering application of continuous compaction control of roadbed [M], Beijing: Science Press, 2016.

[2] Tian Limin. Discussion on Compaction Standard and Acceptance Standard of Railway Subgrade of Passenger Dedicated Line [J]. Railway Standard Design, 2007 (11), pp. $1-4$.

[3] Zhang Jialing, Xu Guanghui, Cai Ying. Study on quality inspection and control of continuous compaction roadbed [J]. Rock and Soil Mechanics, 2015, 36 (04), pp. 1141- 1146.

[4] Minnesota Department of Transportation. Mn/DOT Specification 2106 Pilot Specification for Embankment Grading Materials [S]. St Paul: Minnesota Department of Transportation Office of Research Services, 2007.

[5] Michael A. Mooney, Robert V. Rinehart, Norman W. Facas, et al. "Intelligent Soil Compaction Systems." National Cooperative Highway Research Program Report 676 [R]. Washington, D. C.: Transportation Research Board, 2010.

[6] Fan Juan, Song Xiaodong, Tian Lifeng, Nie Zhihong. Analysis of influencing factors of continuous compaction quality inspection index CMV for high-speed railway subgrade [J]. Journal of Railway Science and Engineering, 2015, 12 (03), pp. 463-467.
[7] Anderegg, R., K. Kaufmann. "Intelligent Compaction with Vibratory Rollers.” Transportation Research Record 1868 [R]. Washington, D. C.: Transportation Research Board, 2004.

[8] Petersen, L. Continuous Compaction Control MnROAD Demonstration. Final report submitted to Mn/DOT, Report No. MN/RC-2005-07 [R]. St Paul: Minnesota Department of Transportation, 2005.

[9] XU Guang-hui, YAN Ze-hua. Study on the Limitation of Harmonic Ratio Index in the Method of Compaction in Continuous Compaction Control Technology [J]. Road Machinery \& Construction Mechanization, 2015, 32 (08), pp. $39-42$.

[10] Thumer H, Sandstrom A. Continuous compaction control, CCC [C]//European Workshop Compaction of Soils and Granular Materials, Presses Fonts et Chaussees, Paris, France. 2000, pp. 237-246.

[11] Nohse Y, Kitano M. Development of a new type of single drum vibratory roller $[\mathrm{C}] / /$ Proc. 14th Intl. Conf. of the Intl. Soc. For Terrain-Vehicle Systems, Vicksburg, MS. 2002, pp. 1 -10.

[12] Krober W, Floss R, Wallrath W. Dynamic soil stiffness as quality criterion for soil compaction [J]. Geotechnics for roads, rail tracks, and earth structures, 2001, pp. 188-199.

[13] Zhang Jialing. Study on the uniformity of support stiffness of continuously compacted ballastless track subgrade structure [D]. Southwest Jiaotong University, 2014.

[14] China Railway Corporation. Q/CR 9210-2015 Technical Specification for Continuous Compaction Control of Railway Subgrade Filling Project [S]. Beijing: China Railway Publishing House, 2017.

[15] Ministry of Transport of the People's Republic of China. JT/T 1127-2017 Technical Conditions for Continuous Compaction Control System for Highway Subgrade Filling Project [S]. Beijing: China Communications Press, 2017.

[16] European Committee for Standardization. PD CEN/TS 17006:2016 Earthworks Continuous Compaction Con- trol [S]. London: BSI Standards Limited, 2017. 